\title{
Boundedness of Singular Integrals on Hardy Type Spaces Associated with Schrödinger Operators
}

\author{
Jianfeng Dong, ${ }^{1}$ Jizheng Huang, ${ }^{2}$ and Heping Liu ${ }^{3}$ \\ ${ }^{1}$ Department of Mathematics, Shanghai University, Shanghai 200444, China \\ ${ }^{2}$ College of Sciences, North China University of Technology, Beijing 100144, China \\ ${ }^{3}$ LMAM, School of Mathematical Sciences, Peking University, Beijing 100871, China
}

Correspondence should be addressed to Jizheng Huang; hjzheng@163.com

Received 19 May 2014; Accepted 21 August 2014

Academic Editor: Jose Luis Sanchez

Copyright (C) 2015 Jianfeng Dong et al. This is an open access article distributed under the Creative Commons Attribution License, which permits unrestricted use, distribution, and reproduction in any medium, provided the original work is properly cited.

Let $L=-\Delta+V$ be a Schrödinger operator on $\mathbb{R}^{n}, n \geq 3$, where $V \not \equiv \quad 0$ is a nonnegative potential belonging to the reverse Hölder class $B_{n / 2}$. The Hardy type spaces $H_{L}^{p}, n /(n+\delta)<p \leq 1$, for some $\delta>0$, are defined in terms of the maximal function with respect to the semigroup $\left\{e^{-t L}\right\}_{t>0}$. In this paper, we investigate the bounded properties of some singular integral operators related to $L$, such as $L^{i \gamma}$ and $\nabla L^{-1 / 2}$, on spaces $H_{L}^{p}$. We give the molecular characterization of $H_{L}^{p}$, which is used to establish the $H_{L}^{p}$-boundedness of singular integrals.

\section{Introduction}

Let $L=-\Delta+V$ be a Schrödinger operator on $\mathbb{R}^{n}, n \geq 3$, where $V \not \equiv \quad 0$ is a nonnegative potential belonging to the reverse Hölder class $B_{q}$ for some $q \geq n / 2$; that is, there exists a constant $C>0$ such that the reverse Hölder inequality

$$
\left(\frac{1}{|B|} \int_{B} V^{q}(x) d x\right)^{1 / q} \leq C\left(\frac{1}{|B|} \int_{B} V(x) d x\right)
$$

holds for every ball $B$ in $\mathbb{R}^{n}$. It is well known that if $V \in B_{q}$ then $V \in B_{q+\varepsilon}$ for some $\varepsilon>0$. Also obviously, $B_{q_{1}} \subset B_{q_{2}}$ when $q_{1}>q_{2}$.

Some singular integral operators related to $L$, such as the imaginary power $L^{i \gamma}$, and the Riesz transform $\nabla L^{-1 / 2}$ have been studied by Shen [1]. Some of his results are following. The operator $L^{i \gamma}$ is a Calderón-Zygmund operator for any $\gamma \in \mathbb{R} . \nabla L^{-1 / 2}$ is a Calderón-Zygmund operator if $q \geq n$. When $n / 2 \leq q<n, \nabla L^{-1 / 2}$ is bounded on $L^{p}$ for $1<p \leq p_{0}$, where $1 / p_{0}=1 / q-1 / n$. The above range of $p$ is optimal. Earlier results were given by Fefferman [2] and Zhong [3].

The Hardy type spaces $H_{L}^{p}, n /(n+\delta)<p \leq 1$ for some $\delta>0$, associated with $L$, are studied by Dziubański and
Zienkiewicz $[4,5]$. They establish the $H_{L}^{p, \infty}$ atomic decomposition theorem and the Riesz transform characterization of $H_{L}^{1}$. Specifically, $\nabla L^{-1 / 2}$ is bounded from $H_{L}^{1}$ to $L^{1}$. We will investigate the bounded properties of the operators $L^{i \gamma}$ and $\nabla L^{-1 / 2}$ on spaces $H_{L}^{p}$. To do this, we give the molecular characterization of $H_{L}^{p}$.

Without loss of generalization, we assume that $V \in B_{q_{0}}$ for some $q_{0}>n / 2$ and set $\delta=\min \left(2-n / q_{0}, 1\right)$. When $q_{0}>n$, we set $\eta=1-n / q_{0}$. Throughout the paper, we will use $A$ and $C$ to denote the positive constants, which are independent of main parameters and may be different at each occurrence. By $B_{1} \sim B_{2}$, we mean that there exists a constant $C>1$ such that $1 / C \leq B_{1} / B_{2} \leq C$.

Let $\left\{T_{t}^{L}\right\}_{t>0}=\left\{e^{-t L}\right\}_{t>0}$ be the semigroup of linear operators generated by $-L$ and $K_{t}^{L}(x, y)$ their kernels. Since $V$ is nonnegative, the Feynman-Kac formula implies that

$$
0 \leq K_{t}^{L}(x, y) \leq K_{t}(x-y)=(4 \pi t)^{-n / 2} e^{-(4 t)^{-1}|x-y|^{2}},
$$

where $K_{t}(x)$ is the convolution kernels of the heat semigroup $\left\{T_{t}\right\}_{t>0}=\left\{e^{t \Delta}\right\}_{t>0}$. The estimate (2) can be improved as 
follows. We introduce the auxiliary function $\rho(x, V)=\rho(x)$ defined by

$$
\rho(x)=\sup \left\{r>0: \frac{1}{r^{n-2}} \int_{B(x, r)} V(y) d y \leq 1\right\} .
$$

It is known that $0<\rho(x)<\infty$. For every $N>0$,

$$
K_{t}^{L}(x, y) \leq C_{N} t^{-n / 2} e^{-(5 t)^{-1}|x-y|^{2}}\left(1+\frac{\sqrt{t}}{\rho(x)}+\frac{\sqrt{t}}{\rho(y)}\right)^{-N},
$$

(cf. [6, Theorem 4.10]). Let $0<\delta^{\prime}<\delta$; for every $N>0$ and all $|h| \leq \sqrt{t}$

$$
\begin{aligned}
& \left|K_{t}^{L}(x+h, y)-K_{t}^{L}(x, y)\right| \\
& \quad \leq C_{N}\left(\frac{|h|}{\sqrt{t}}\right)^{\delta^{\prime}} t^{-n / 2} e^{-A t^{-1}|x-y|^{2}}\left(1+\frac{\sqrt{t}}{\rho(x)}+\frac{\sqrt{t}}{\rho(y)}\right)^{-N}
\end{aligned}
$$

(cf. [6, Proposition 4.11]).

We define the Hardy type spaces $H_{L}^{p}, n /(n+\delta)<p \leq 1$, in terms of the maximal function with respect to the semigroup $\left\{T_{t}^{L}\right\}_{t>0}$.

For $p=1$, the Hardy space $H_{L}^{1}$ is defined, according to Dziubański and Zienkiewicz [4], by

$$
H_{L}^{1}=\left\{f \in L^{1}: M^{L} f \in L^{1}\right\},
$$

where

$$
M^{L} f(x)=\sup _{t>0}\left|T_{t}^{L} f(x)\right| .
$$

The norm of a function $f \in H_{L}^{1}$ is defined to be $\|f\|_{H_{L}^{1}}=$ $\left\|M^{L} f\right\|_{L^{1}}$.

The Hardy spaces, $H_{L}^{p}, n /(n+\delta)<p<1$, consist of some kind of distributions. But $M^{L} f(x)$ may have no meaning for a tempered distribution $f$ because $K_{t}^{L}(x, y)$ are not smooth. Let $f$ be a locally integrable function. $B=B(x, r)$ is the ball of radius $r$ centered at $x$. Set

$$
\begin{aligned}
f_{B} & =\frac{1}{|B|} \int_{B} f(y) d y, \\
f(B, V) & = \begin{cases}f_{B}, & \text { if } r<\rho(x), \\
0, & \text { if } r \geq \rho(x) .\end{cases}
\end{aligned}
$$

Let $n /(n+\delta)<p<1,1 \leq q^{\prime} \leq \infty$. A locally integrable function $f$ is said to be in the Campanato type space $\Lambda_{1 / p-1, q^{\prime}}^{L}$ if

$$
\begin{aligned}
\|f\|_{\Lambda_{1 / p-1, q^{\prime}}^{L}} & =\sup _{B \subset \mathbb{R}^{d}}\left\{|B|^{1-1 / p}\left(\int_{B}|f-f(B, V)|^{q^{\prime}} \frac{d x}{|B|}\right)^{1 / q^{\prime}}\right\} \\
& <\infty .
\end{aligned}
$$

All spaces $\Lambda_{1 / p-1, q^{\prime}}^{L}$ are mutually coincident with equivalent norms and will be simply denoted by $\Lambda_{1 / p-1}^{L}$ (cf. [7]). Due to (4) and (5), for every $t>0$,

$$
\sup _{x \in \mathbb{R}^{d}}\left\|K_{t}^{L}(x, \cdot)\right\|_{\Lambda_{1 / p-1}^{L}}<C t^{-d / 2 p}
$$

(cf. [7, Lemma 1]). Thus the semigroup maximal function $M f$ is well defined for distributions in $\left(\Lambda_{1 / p-1}^{L}\right)^{*}$. We define the Hardy space, $H_{L}^{p}, n /(n+\delta)<p<1$, by

$$
H_{L}^{p}=\left\{f \in\left(\Lambda_{1 / p-1}^{L}\right)^{*}: M^{L} f \in L^{p}\right\},
$$

and set $\|f\|_{H_{L}^{p}}=\left\|M^{L} f\right\|_{L^{p}}$

Similar to the classical case, the Hardy space $H_{L}^{p}$ admits an atomic decomposition. Let $n /(n+\delta)<p \leq 1 \leq q \leq \infty, p \neq$ $q$. A function $a$ is called an $H_{L}^{p, q}$-atom associated with a ball $B\left(x_{0}, r\right)$ if

(1) supp $a \subset B\left(x_{0}, r\right)$,

(2) $\|a\|_{L^{q}} \leq\left|B\left(x_{0}, r\right)\right|^{1 / q-1 / p}$,

(3) if $r<\rho\left(x_{0}\right)$, then $\int a(x) d x=0$.

Proposition 1 (see [7, Theorem 1]). Given p, q as above, then $f \in H_{L}^{p}$ if and only if $f$ can be written as $f=\sum_{j} \lambda_{j} a_{j}$, where $a_{j}$ are $H_{L}^{p, q}$-atoms and $\sum_{j}\left|\lambda_{j}\right|^{p}<\infty$. The sum converges in $H_{L}^{p}$ norm and also in $\left(\Lambda_{1 / p-1}^{L}\right)^{*}$ when $p<1$. Moreover,

$$
\|f\|_{H_{L}^{p}} \sim\|f\|_{H_{L}^{p, q, a}}=\inf \left\{\left(\sum_{j}\left|\lambda_{j}\right|^{p}\right)^{1 / p}\right\},
$$

where the infimum is taken over all decompositions of $f$ into $H_{L}^{p, q}$-atoms.

Now we state the main results in this paper.

Theorem 2. For any $\gamma \in \mathbb{R}$, the imaginary power $L^{i \gamma}$ is bounded on $H_{L}^{p}$ for $n /(n+\delta)<p \leq 1$. When $q_{0}>n$, the Riesz transform $\nabla L^{-1 / 2}$ is bounded on $H_{L}^{p}$ for $n /(n+\eta)<p \leq 1$. Moreover, $\nabla L^{-1 / 2}$ is bounded on $H_{L}^{1}$ whenever $q_{0}>n / 2$.

Remark 3. When $n / 2<q_{0}<n$, the kernel of Riesz transform $\nabla L^{-1 / 2}$ only satisfies the Hörmander condition with respect to the second variable, which is weaker than the smoothness condition of standard kernels. Thus we cannot expect, in general consideration, to deal with the boundedness of $\nabla L^{-1 / 2}$ for the case of $p<1$.

In order to prove Theorem 2, we give the molecular characterization of $H_{L}^{p}$.

Let $n /(n+\delta)<p \leq 1 \leq q \leq \infty, p \neq q$ and $\epsilon>1 / p-1$. Set $a=1-1 / p+\epsilon, b=1-1 / q+\epsilon$. A function $M \in L^{q}$ is called an $H_{L}^{p, q, \epsilon}$-molecule with the center $x_{0}$ if

(1) $|x|^{n b} M(x) \in L^{q}$, 
(2) $\mathcal{N}(M)=\mu_{1}^{b-a}\|M\|_{L^{q}}^{a / b}\left\|\left|\cdot-x_{0}\right|^{n b} M\right\|_{L^{q}}^{1-a / b} \leq 1$,

(3) if $\|M\|_{L^{q}}^{1 /(a-b)}<\mu_{1} \rho\left(x_{0}\right)^{n}$, then $\int M(x) d x=0$,

where $\mu_{1}$ is the volume of the unit ball.

Theorem 4. Given $p, q, \epsilon$ as above, then $f \in H_{L}^{p}$ if and only if $f$ can be written as $f=\sum_{j} \lambda_{j} M_{j}$, where $M_{j}$ are $H_{L}^{p, q, \epsilon}$-molecules and $\sum_{j}\left|\lambda_{j}\right|^{p}<\infty$. The sum converges in $H_{L}^{p}$ norm and also in $\left(\Lambda_{1 / p-1}^{L}\right)^{*}$ when $p<1$, where $M_{j}$ are $H_{L^{-}}^{p}$ molecules. Moreover,

$$
\|f\|_{H_{L}^{p}} \sim\|f\|_{H_{L}^{p, q, \epsilon, M}}=\inf \left\{\left(\sum_{j}\left|\lambda_{j}\right|^{p}\right)^{1 / p}\right\}
$$

where the infimum is taken over all decompositions of $f$ into $H_{L}^{p, q, \epsilon}$-molecules.

Remark 5. It is easy to verify that any $H_{L}^{p, q}$-atom is an $H_{L}^{p, q, \epsilon}$ molecule with a constant factor less than or equal to 1 . We will see that the image of an $H_{L}^{p, q}$-atom under the action of a singular integral operator may not be an $H_{L}^{p, q, \epsilon}$-molecule but is a sum of two $H_{L}^{p, q, \epsilon}$-molecules up to constant factors. This is different from the classical case.

This paper is organized as follows. In Section 2, we collect some useful facts and results about the potential $V$, the auxiliary function $\rho(x)$ and the kernels of operators $L^{i \gamma}$, and $\nabla L^{-1 / 2}$, which will be used in the sequel. Most of these results are already known. In Section 3, we prove Theorem 4 . The proof of Theorem 2 is given in the last two sections. The $H_{L}^{p}-$ boundedness for $p<1$ is proved in Section 4 while $H_{L^{-}}^{1}$ boundedness is proved in Section 5 .

\section{Preliminaries}

First we list some known facts and results about the potential $V$, the auxiliary function $\rho(x)$, and the kernels of operators $L^{i \gamma}$ and $\nabla L^{-1 / 2}$.

Lemma 6. $V(x) d x$ is a doubling measure; that is, there exists a constant $C_{0}>0$ such that

$$
\int_{B(x, 2 r)} V(y) d y \leq C_{0} \int_{B(x, r)} V(y) d y .
$$

Lemma 7. Consider

$$
\begin{array}{r}
\frac{1}{r^{n-2}} \int_{B(x, r)} V(y) d y \leq C\left(\frac{R}{r}\right)^{n / q_{0}-2} \frac{1}{R^{n-2}} \int_{B(x, R)} V(y) d y \\
0<r<R<\infty .
\end{array}
$$

Lemma 8. There exists $m_{0}>0$ such that

$$
\frac{1}{R^{n-2}} \int_{B(x, R)} V(y) d y \leq C\left(1+\frac{R}{\rho(x)}\right)^{m_{0}} .
$$

Lemma 9. There exists $k_{0}>0$ such that

$$
\frac{1}{C}\left(1+\frac{|x-y|}{\rho(x)}\right)^{-k_{0}} \leq \frac{\rho(y)}{\rho(x)} \leq C\left(1+\frac{|x-y|}{\rho(x)}\right)^{k_{0} /\left(k_{0}+1\right)} .
$$

In particular, $\rho(y) \sim \rho(x)$ if $|x-y|<C \rho(x)$.

Let $F_{\gamma}^{L}(x, y)$ and $F_{\gamma}(x, y)$ be the kernels of $L^{i \gamma}$ and $(-\Delta)^{i \gamma}$, respectively, and $R^{L}(x, y)$ and $R(x, y)$ the kernels of $\nabla L^{-1 / 2}$ and $\nabla(-\Delta)^{-1 / 2}$, respectively. Set $\widetilde{F}_{\gamma}(x, y)=F_{\gamma}^{L}(x, y)-$ $F_{\gamma}(x, y), \widetilde{R}(x, y)=R^{L}(x, y)-R(x, y)$.

Lemma 10. $L^{i \gamma}$ is a Calderón-Zygmund operator. It does not matter to assume that $n / 2<q_{0}<n$. The kernel $F_{\gamma}^{L}(x, y)$ satisfies

$$
\left|F_{\gamma}^{L}(x, y+h)-F_{\gamma}^{L}(x, y)\right| \leq \frac{C e^{\pi|\gamma| / 2}|h|^{\delta}}{|x-y|^{n+\delta}}, \quad|h| \leq \frac{|x-y|}{2},
$$

and, for any $N>0$,

$$
\left|F_{\gamma}^{L}(x, y)\right| \leq \frac{C_{N} e^{\pi|\gamma| / 2}}{|x-y|^{n}}\left(1+\frac{|x-y|}{\rho(y)}\right)^{-N}
$$

In addition,

$$
\left|\widetilde{F}_{\gamma}(x, y)\right| \leq \frac{C e^{\pi|\gamma| / 2}}{|x-y|^{n}}\left(\frac{|x-y|}{\rho(y)}\right)^{\delta} .
$$

Lemma 11. When $n / 2<q_{0}<n, \nabla L^{-1 / 2}$ is bounded on $L^{p}$ for $1<p \leq p_{0}$, where $1 / p_{0}=1 / q_{0}-1 / n$. The kernel $R^{L}(x, y)$ satisfies, for any $N>0$,

$$
\begin{aligned}
\left|R^{L}(x, y)\right| \leq & \frac{C_{N}}{|x-y|^{n-1}}\left(\int_{B(x,|x-y| / 4)} \frac{V(z) d z}{|z-x|^{n-1}}+\frac{1}{|x-y|}\right) \\
& \times\left(1+\frac{|x-y|}{\rho(y)}\right)^{-N} .
\end{aligned}
$$

In addition,

$$
\begin{aligned}
& |\widetilde{R}(x, y)| \\
& \leq \frac{C}{|x-y|^{n-1}} \\
& \quad \times\left(\int_{B(x,|x-y| / 4)} \frac{V(z) d z}{|z-x|^{n-1}}+\frac{1}{|x-y|}\left(\frac{|x-y|}{\rho(y)}\right)^{\delta}\right) .
\end{aligned}
$$


Lemma 12. When $q_{0}>n, \nabla L^{-1 / 2}$ is a Calderón-Zygmund operator. The kernel $R^{L}(x, y)$ satisfies

$$
\begin{array}{r}
\left|R^{L}(x, y+h)-R^{L}(x, y)\right| \leq \frac{C|h|^{\eta}}{|x-y|^{n+\eta}}, \\
|h| \leq \frac{|x-y|}{2},
\end{array}
$$

and, for any $N>0$,

$$
\left|R^{L}(x, y)\right| \leq \frac{C_{N}}{|x-y|^{n}}\left(1+\frac{|x-y|}{\rho(y)}\right)^{-N} .
$$

In addition, for any $\delta^{\prime}<1$,

$$
|\widetilde{R}(x, y)| \leq \frac{C}{|x-y|^{n}}\left(\frac{|x-y|}{\rho(y)}\right)^{\delta^{\prime}} .
$$

For Lemmas 6-12, we refer readers to [1]. We also need the following estimates about $\widetilde{F}_{\gamma}(x, y)$ and $\widetilde{R}(x, y)$.

Lemma 13. When $n / 2<q_{0}<n$,

$$
\begin{array}{r}
\left|\widetilde{F}_{\gamma}(x, y+h)-\widetilde{F}_{\gamma}(x, y)\right| \leq \frac{C e^{\pi|\gamma| / 2}}{|x-y|^{n}}\left(\frac{|h|}{\rho(y)}\right)^{\delta}, \\
|h| \leq \frac{|x-y|}{2} .
\end{array}
$$

When $q_{0}>n$, for any $\delta^{\prime}<1$,

$$
\begin{array}{r}
|\widetilde{R}(x, y+h)-\widetilde{R}(x, y)| \leq \frac{C}{|x-y|^{n}}\left(\frac{|h|}{\rho(y)}\right)^{\delta^{\prime}}, \\
|h| \leq \frac{|x-y|}{2} .
\end{array}
$$

Proof. It is well known that

$$
\begin{array}{r}
\left|F_{\gamma}(x, y+h)-F_{\gamma}(x, y)\right| \leq \frac{C|h|}{|x-y|^{n+1}}, \\
|h| \leq \frac{|x-y|}{2}, \\
|R(x, y+h)-R(x, y)| \leq \frac{C|h|}{|x-y|^{n+1}}, \\
|h| \leq \frac{|x-y|}{2} .
\end{array}
$$

Therefore, we also have the estimates

$$
\begin{gathered}
\left|\widetilde{F}_{\gamma}(x, y+h)-\widetilde{F}_{\gamma}(x, y)\right| \leq \frac{C e^{\pi|\gamma| / 2}|h|^{\delta}}{|x-y|^{n+\delta}}, \quad|h| \leq \frac{|x-y|}{2}, \\
|\widetilde{R}(x, y+h)-\widetilde{R}(x, y)| \leq \frac{C|h|^{\eta}}{|x-y|^{n+\eta}}, \quad|h| \leq \frac{|x-y|}{2} .
\end{gathered}
$$

We may assume that $|x-y|<\rho(y)$. Otherwise, Lemma 13 is obvious.

We will use the following known facts (cf. [1]). Let $\Gamma^{L}(x, y, \tau)$ and $\Gamma(x, y, \tau)$ denote, respectively, the fundamental solutions for the operators $L+i \tau$ and $-\Delta+i \tau$ in $\mathbb{R}^{n}$, where $\tau \in \mathbb{R}$. They satisfy the following estimates. For any $k>0$ and $|h| \leq|x-y| / 2$,

$$
\begin{gathered}
|\Gamma(x, y, \tau)| \leq \frac{C_{k}}{\left(1+|\tau|^{1 / 2}|x-y|\right)^{k}} \frac{1}{|x-y|^{n-2}}, \\
\left|\Gamma^{L}(x, y+h, \tau)-\Gamma^{L}(x, y, \tau)\right| \\
\leq \frac{C_{k}}{\left(1+|\tau|^{1 / 2}|x-y|\right)^{k}} \frac{|h|^{\delta}}{|x-y|^{n-2+\delta}}\left(1+\frac{|x-y|}{\rho(y)}\right)^{-k}
\end{gathered}
$$

when $n / 2<q_{0}<n$. Set $\widetilde{\Gamma}(x, y, \tau)=\Gamma^{L}(x, y, \tau)-\Gamma(x, y, \tau)$. Then $\widetilde{\Gamma}(x, y, \tau)$ is expressed as

$$
\widetilde{\Gamma}(x, y, \tau)=-\int_{\mathbb{R}^{n}} \Gamma(x, z, \tau) V(z) \Gamma^{L}(z, y, \tau) d z .
$$

Thus,

$$
\begin{aligned}
& |\widetilde{\Gamma}(x, y+h, \tau)-\widetilde{\Gamma}(x, y, \tau)| \\
& \leq \int_{\mathbb{R}^{n}}|\Gamma(x, z, \tau)| V(z)\left|\Gamma^{L}(z, y+h, \tau)-\Gamma^{L}(z, y, \tau)\right| d z \\
& \leq \int_{\mathbb{R}^{n}}\left(C_{k}|h|^{\delta} V(z)\left(1+\rho(y)^{-1}|z-y|\right)^{-k} d z\right) \\
& \quad \times\left(\left(1+|\tau|^{1 / 2}|x-z|\right)^{k}\left(1+|\tau|^{1 / 2}|z-y|\right)^{k}\right. \\
& \left.\quad \quad \times|x-z|^{n-2}|z-y|^{n-2+\delta}\right)^{-1} \\
& =\int_{|z-x|<|x-y| / 2}(\cdots)+\int_{|z-y|<|x-y| / 2}(\cdots) \\
& \quad+\int_{|z-x| \geq|x-y| / 2,|z-y| \geq|x-y| / 2}(\cdots) \\
& =I_{1}+I_{2}+I_{3} .
\end{aligned}
$$

Note that $V \in B_{q_{0}+\varepsilon}$ for some $\varepsilon>0$. Using Hölder inequality and $B_{q_{0}+\varepsilon}$ condition, it is easy to see that, for $0 \leq \sigma \leq \delta$,

$$
\int_{B(x, R)} \frac{V(y)}{|x-y|^{n-2+\sigma}} d y \leq \frac{C}{R^{n-2+\sigma}} \int_{B(x, R)} V(y) d y .
$$


Note that $\rho(x) \sim \rho(y)$ when $|x-y|<\rho(y)$. Making use of (34), we get

$$
\begin{aligned}
I_{1} \leq & \frac{C_{k}|h|^{\delta}}{\left(1+|\tau|^{1 / 2}|x-y|\right)^{k}|x-y|^{n-2+\delta}} \int_{|z-x|<|x-y| / 2} \frac{V(z) d z}{|x-z|^{n-2}} \\
\leq & \frac{C_{k}|h|^{\delta}}{\left(1+|\tau|^{1 / 2}|x-y|\right)^{k}|x-y|^{n-2+\delta}} \frac{1}{|x-y|^{n-2}} \\
& \times \int_{|z-x|<|x-y| / 2} V(z) d z \\
\leq & \frac{C_{k}}{\left(1+|\tau|^{1 / 2}|x-y|\right)^{k}|x-y|^{n-2}}\left(\frac{|h|}{\rho(y)}\right)^{\delta},
\end{aligned}
$$

where we have used Lemma 7 in the last inequality. Similarly,

$$
\begin{aligned}
I_{2} \leq & \frac{C_{k}|h|^{\delta}}{\left(1+|\tau|^{1 / 2}|x-y|\right)^{k}|x-y|^{n-2}} \int_{|z-y|<|x-y| / 2} \frac{V(z) d z}{|z-y|^{n-2+\delta}} \\
\leq & \frac{C_{k}|h|^{\delta}}{\left(1+|\tau|^{1 / 2}|x-y|\right)^{k}|x-y|^{n-2+\delta}} \frac{1}{|x-y|^{n-2}} \\
& \times \int_{|z-y|<|x-y| / 2} V(z) d z \\
\leq & \frac{C_{k}}{\left(1+|\tau|^{1 / 2}|x-y|\right)^{k}|x-y|^{n-2}}\left(\frac{|h|}{\rho(y)}\right)^{\delta} .
\end{aligned}
$$

To estimate $I_{3}$, we write

$$
\begin{aligned}
I_{3} \leq & \frac{C_{k}|h|^{\delta}}{\left(1+|\tau|^{1 / 2}|x-y|\right)^{k}} \\
& \times \int_{|z-y| \geq|x-y| / 2}\left(1+\frac{|x-y|}{\rho(y)}\right)^{-k} \frac{V(z) d z}{|z-y|^{2 n-4+\delta}} \\
\leq & \frac{C_{k}|h|^{\delta}}{\left(1+|\tau|^{1 / 2}|x-y|\right)^{k}} \\
& \times\left(\int_{|x-y| / 2 \leq|z-y|<\rho(y)} \frac{V(z) d z}{|z-y|^{2 n-4+\delta}}\right. \\
& \left.+\rho(y)^{k} \int_{|z-y| \geq \rho(y)} \frac{V(z) d z}{|z-y|^{2 n-4+\delta+k}}\right) .
\end{aligned}
$$

Using Hölder inequality and $B_{q_{0}}$ condition, we obtain

$$
\begin{aligned}
& \int_{|x-y| / 2 \leq|z-y|<\rho(y)} \frac{V(z) d z}{|z-y|^{2 n-4+\delta}} \\
& \leq\left(\int_{|z-y|<\rho(y)} V(z)^{q_{0}} d z\right)^{1 / q_{0}} \\
& \quad \times\left(\int_{|z-y| \geq|x-y| / 2} \frac{d z}{|z-y|^{(2 n-4+\delta) q_{0}^{\prime}}}\right)^{1 / q_{0}^{\prime}} \\
& \leq \frac{C}{|x-y|^{n-2} \rho(y)^{\delta}} .
\end{aligned}
$$

Using Lemma 6 and taking $k$ sufficiently large, we get

$$
\begin{aligned}
& \rho(y)^{k} \int_{|z-y| \geq \rho(y)} \frac{V(z) d z}{|z-y|^{2 n-4+\delta+k}} \\
& \leq C \rho(y)^{4-2 n-\delta} \sum_{j=1}^{\infty} 2^{-j(2 n-4+\delta+k)} \int_{|z-y| \leq 2^{j} \rho(y)} V(z) d z \\
& \leq C \rho(y)^{4-2 n-\delta} \sum_{j=1}^{\infty} 2^{-j(2 n-4+\delta+k)} C_{0}^{j} \int_{|z-y| \leq \rho(y)} V(z) d z \\
& \leq C \rho(y)^{2-n-\delta} \\
& \leq \frac{C}{|x-y|^{n-2} \rho(y)^{\delta}} .
\end{aligned}
$$

Therefore,

$$
\begin{aligned}
& |\widetilde{\Gamma}(x, y+h, \tau)-\widetilde{\Gamma}(x, y, \tau)| \\
& \quad \leq \frac{C_{k}}{\left(1+|\tau|^{1 / 2}|x-y|\right)^{k}|x-y|^{n-2}}\left(\frac{|h|}{\rho(y)}\right)^{\delta} .
\end{aligned}
$$

We also have

$$
\nabla_{x} \widetilde{\Gamma}(x, y, \tau)=-\int_{\mathbb{R}^{n}} \nabla_{x} \Gamma(x, z, \tau) V(z) \Gamma^{L}(z, y, \tau) d z,
$$

where $\nabla_{x} \Gamma(x, z, \tau)$ satisfies the estimate

$$
\left|\nabla_{x} \Gamma(x, y, \tau)\right| \leq \frac{C_{k}}{\left(1+|\tau|^{1 / 2}|x-y|\right)^{k}} \frac{1}{|x-y|^{n-1}} .
$$

If $q_{0}>n$, by the same argument as (40), for any $\delta^{\prime}<1$,

$$
\begin{aligned}
& \left|\nabla_{x} \widetilde{\Gamma}(x, y+h, \tau)-\nabla_{x} \widetilde{\Gamma}(x, y, \tau)\right| \\
& \quad \leq \frac{C_{k}}{\left(1+|\tau|^{1 / 2}|x-y|\right)^{k}|x-y|^{n-1}}\left(\frac{|h|}{\rho(y)}\right)^{\delta^{\prime}} .
\end{aligned}
$$


By the functional calculus and making use of (40), we obtain

$$
\begin{aligned}
& \left|\widetilde{F}_{\gamma}(x, y+h)-\widetilde{F}_{\gamma}(x, y)\right| \\
& \quad=\frac{1}{2 \pi}\left|\int_{\mathbb{R}}(-i \tau)^{i \gamma}(\widetilde{\Gamma}(x, y+h, \tau)-\widetilde{\Gamma}(x, y, \tau)) d \tau\right| \\
& \quad \leq \frac{C e^{\pi|\gamma| / 2}}{|x-y|^{n}}\left(\frac{|h|}{\rho(y)}\right)^{\delta} .
\end{aligned}
$$

This proves (26).

Similarly, it follows from (43) that

$$
\begin{aligned}
& |\widetilde{R}(x, y+h)-\widetilde{R}(x, y)| \\
& \quad=\frac{1}{2 \pi}\left|\int_{\mathbb{R}}(-i \tau)^{-1 / 2}\left(\nabla_{x} \widetilde{\Gamma}(x, y+h, \tau)-\nabla_{x} \widetilde{\Gamma}(x, y, \tau)\right) d \tau\right| \\
& \quad \leq \frac{C}{|x-y|^{n}}\left(\frac{|h|}{\rho(y)}\right)^{\delta^{\prime}} .
\end{aligned}
$$

This proves (27).

\section{Molecular Characterization}

Essentially, the proof of Theorem 4 is the same as the usual molecular theory.

Proof of Theorem 4. By Proposition 1, it is sufficient to prove that for any $H_{L}^{p, q, \epsilon}$-molecule $M(x)$ admits an atomic decomposition $M=\sum_{j} \lambda_{j} a_{j}$, where $a_{j}$ are $H_{L}^{p, q}$-atoms and $\sum_{j}\left|\lambda_{j}\right|^{p}<C$

We will give the proof in case $q=2$. The proof is similar in the case of $q \neq 2$. Suppose $M(x)$ is an $H_{L}^{p, 2, \epsilon}$-molecule centered at $x_{0}$. Let $\sigma=\|M\|_{L^{2}}^{1 /(a-b)}$, where $\epsilon>1 / p-1, a=$ $1-1 / p+\epsilon, \quad b=1 / 2+\epsilon$. If $\sigma<\mu_{1} \rho\left(x_{0}\right)^{n}$, we return the usual molecular theory (cf. [8]). Thus nothing needs to be proved. Suppose $\sigma \geq \mu_{1} \rho\left(x_{0}\right)^{n}$. Set

$$
\begin{gathered}
B_{k}=\left\{x:\left|x-x_{0}\right| \leq 2^{k} \mu_{1}^{-1 / n} \sigma^{1 / n}\right\}, \quad k=0,1,2, \ldots, \\
E_{0}=B_{0}, \quad E_{k}=B_{k} \backslash B_{k-1}, \quad k=1,2, \ldots .
\end{gathered}
$$

Then

$$
M(x)=\sum_{k=0}^{\infty} M(x) \chi_{E_{k}}(x)=\sum_{k=0}^{\infty} M_{k}(x) .
$$

Note that supp $M_{k} \subset B_{k}$ and $2^{k} \mu_{1}^{-1 / n} \sigma^{1 / n} \geq \rho\left(x_{0}\right), k=$ $0,1,2, \ldots$ Also we have

$$
\begin{aligned}
\left\|M_{0}\right\|_{L^{2}} & \leq\|M\|_{L^{2}}=\sigma^{a-b}=\left|B_{0}\right|^{1 / 2-1 / p}, \\
\left\|M_{k}\right\|_{L^{2}} & \leq 2^{-(k-1) n b} \mu_{1}^{b} \sigma^{-b}\left\|\left|\cdot-x_{0}\right|^{n b} M\right\|_{L^{2}} \\
& \leq 2^{-(k-1) n b} \sigma^{-b}\|M\|_{L^{2}}^{a /(a-b)} \\
& =2^{-(k-1) n b} \sigma^{a-b} \\
& =2^{n b-k n a}\left|B_{k}\right|^{1 / 2-1 / p} .
\end{aligned}
$$

Thus $M_{k}(x)=\lambda_{k} a_{k}(x), k=0,1,2, \ldots$, where $a_{k}$ are $H_{L}^{p, q}$ atoms and $\sum_{k=0}^{\infty}\left|\lambda_{k}\right|^{p}<C$.

Originally, the sum in (47) converges pointwise. When $p=1$, it is easy to see that the sum in (47) converges in $L^{1}$. If $n /(n+\delta)<p<1$, for any $g \in \Lambda_{1 / p-1}^{L}$,

$$
\begin{aligned}
\left\|\left(1+|x|^{n b}\right)^{-1} g\right\|_{L^{2}} & \\
\leq & \left(\int_{|x|<\rho(0)}|g(x)|^{2} d x\right)^{1 / 2}+\sum_{k=1}^{\infty} 2^{-(k-1) n b} \rho(0)^{-n b} \\
& \times\left(\int_{2^{k-1} \rho(0) \leq|x|<2^{k} \rho(0)}|g(x)|^{2} d x\right)^{1 / 2} \\
\leq & C \sum_{k=0}^{\infty} 2^{-k n a}\|g\|_{\Lambda_{1 / p-1}^{L}} \\
\leq & C\|g\|_{\Lambda_{1 / p-1}^{L}} .
\end{aligned}
$$

Therefore,

$$
\|M g\|_{L^{1}} \leq\left\|\left(1+|x|^{n b}\right) M\right\|_{L^{2}}\left\|\left(1+|x|^{n b}\right)^{-1} g\right\|_{L^{2}}<\infty .
$$

It follows that the sum in (47) converges in $\left(\Lambda_{1 / p-1}^{L}\right)^{*}$. The proof of Theorem 4 is completed.

\section{4. $H_{L}^{p}$-Boundedness}

In this section, we prove the boundedness of $L^{i \gamma}$ on $H_{L}^{p}, n /(n+$ $\delta)<p \leq 1$. When $q_{0}>n$, the boundedness of $\nabla L^{-1 / 2}$ on $H_{L}^{p}, n /(n+\eta)<p \leq 1$, can be proved by the same method. In fact, their kernels satisfy similar estimates.

Let $a(x)$ be an $H_{L}^{p, q}$-atom associated with a ball $B\left(x_{0}, r\right)$ for some suitable $q$. If $r \geq \rho\left(x_{0}\right)$, we will prove that $L^{i \gamma} a(x)$ is an $H_{L}^{p, q, \epsilon}$-molecule up to a constant factor. If $r<\rho\left(x_{0}\right)$, $L^{i \gamma} a(x)$ may be not an $H_{L}^{p, q, \epsilon}$-molecule up to a constant factor but $(-\Delta)^{i \gamma} a(x)$ is (cf. [9]). We will prove that $\left(L^{i \gamma}-(-\Delta)^{i \gamma}\right) a(x)$ is an $H_{L}^{p, q, \epsilon}$-molecule up to a constant factor for some suitable $\epsilon$. This means that $\left\|L^{i \gamma} a(x)\right\|_{H_{L}^{p}} \leq C$ uniformly. Because the semigroup maximal function $M^{L} f$ is subadditive, by Proposition $1, L^{i \gamma}$ is bounded on $H_{L}^{p}, \quad n /(n+\delta)<p \leq 1$.

First, let $r \geq \rho\left(x_{0}\right)$. Because

$$
\left\|L^{i \gamma} a(x)\right\|_{L^{q}} \leq C\|a(x)\|_{L^{q}} \leq C\left|B\left(x_{0}, r\right)\right|^{a-b},
$$

where $\epsilon>1 / p-1, a=1-1 / p+\epsilon, b=1-1 / q+\epsilon$, we have

$$
\left\|L^{i \gamma} a(x)\right\|_{L^{q}}^{1 /(a-b)} \geq \frac{1}{C} \rho\left(x_{0}\right)^{n} .
$$


Thus there needs no the cancelation condition. We only need to estimate $\mathcal{N}\left(L^{i \gamma} a\right)$. Write

$$
\begin{aligned}
& \left(\int_{\mathbb{R}^{n}}\left|x-x_{0}\right|^{n b q}\left|L^{i \gamma} a(x)\right|^{q} d x\right)^{1 / q} \\
& \leq\left(\int_{B\left(x_{0}, 2 r\right)}\left|x-x_{0}\right|^{n b q}\left|L^{i \gamma} a(x)\right|^{q} d x\right)^{1 / q} \\
& \quad+\left(\int_{\left|x-x_{0}\right| \geq 2 r}\left|x-x_{0}\right|^{n b q}\left|L^{i \gamma} a(x)\right|^{q} d x\right)^{1 / q} \\
& =I_{1}+I_{2} .
\end{aligned}
$$

It is obvious that

$$
\begin{gathered}
I_{1} \leq r^{n b}\left\|L^{i \gamma} a(x)\right\|_{L^{q}} \leq C\left|B\left(x_{0}, r\right)\right|^{b} \\
\times\|a(x)\|_{L^{q}} \leq C\left|B\left(x_{0}, r\right)\right|^{a} .
\end{gathered}
$$

For $y \in B\left(x_{0}, r\right)$, if $\rho(y)>r$, by Lemma $9, \rho(y) \leq C \rho\left(x_{0}\right) \leq$ $C r$. Note that $|x-y| \sim\left|x-x_{0}\right|$ when $x \notin B\left(x_{0}, 2 r\right), y \in$ $B\left(x_{0}, r\right)$. Using Lemma 10, we get

$$
\begin{aligned}
& I_{2}=\left(\int _ { | x - x _ { 0 } | \geq 2 r } \left(\int_{B\left(x_{0}, r\right)}\left|x-x_{0}\right|^{n b}\right.\right. \\
& \left.\left.\quad \times\left|F_{\gamma}^{L}(x, y) a(y)\right| d y\right)^{q} d x\right)^{1 / q} \\
& \leq \int_{B\left(x_{0}, r\right)}|a(y)| d y\left(\int_{\left|x-x_{0}\right| \geq 2 r}\left|x-x_{0}\right|^{n b q}\right. \\
& \leq C \int_{B\left(x_{0}, r\right)}|a(y)| r_{\gamma}^{N} d y \\
& \left.\quad \times\left.\left(\int_{\left|x-x_{0}\right| \geq 2 r} \mid x-y\right)\right|^{q} d x\right)^{1 / q} \\
& \leq C r^{n-n / p+N} r^{n b-n / q^{\prime}-N} \\
& \leq C\left|B\left(x_{0}, r\right)\right|^{a}
\end{aligned}
$$

and provide $N>n \epsilon$. Therefore,

$$
\left\|\left|x-x_{0}\right|^{n b} L^{i \gamma} a(x)\right\|_{L^{q}} \leq C\left|B\left(x_{0}, r\right)\right|^{a}
$$

It follows that

$$
\mathcal{N}\left(L^{i \gamma} a\right)=\mu_{1}^{b-a}\left\|L^{i \gamma} a(x)\right\|_{L^{q}}^{a / b}\left\|\left|x-x_{0}\right|^{n b} L^{i \gamma} a(x)\right\|_{L^{q}}^{1-a / b} \leq C .
$$

Next, suppose $r<\rho\left(x_{0}\right)$. Let us estimate $\left\|\left(L^{i \gamma}-(-\Delta)^{i \gamma}\right) a(x)\right\|_{L^{q}}$. Consider

$$
\begin{aligned}
\left(\int_{\mathbb{R}^{n}} \mid\right. & \left.\left.\left(L^{i \gamma}-(-\Delta)^{i \gamma}\right) a(x)\right|^{q} d x\right)^{1 / q} \\
\leq & \left(\int_{B\left(x_{0}, 2 r\right)}\left|\left(L^{i \gamma}-(-\Delta)^{i \gamma}\right) a(x)\right|^{q} d x\right)^{1 / q} \\
& +\left(\int_{2 r \leq\left|x-x_{0}\right|<2 \rho\left(x_{0}\right)}\left|\left(L^{i \gamma}-(-\Delta)^{i \gamma}\right) a(x)\right|^{q} d x\right)^{1 / q} \\
& +\left(\int_{\left|x-x_{0}\right| \geq 2 \rho\left(x_{0}\right)}\left|\left(L^{i \gamma}-(-\Delta)^{i \gamma}\right) a(x)\right|^{q} d x\right)^{1 / q} \\
= & J_{1}+J_{2}+J_{3} .
\end{aligned}
$$

Note that $\rho(y) \sim \rho\left(x_{0}\right)$, when $y \in B\left(x_{0}, r\right)$ and by Lemma 10 , we have

$J_{1}$

$$
\begin{aligned}
& =\left(\int_{B\left(x_{0}, 2 r\right.}\left(\int_{B\left(x_{0}, r\right)}\left|\widetilde{F}_{\gamma}(x, y) a(y)\right| d y\right)^{q} d x\right)^{1 / q} \\
& \leq \int_{B\left(x_{0}, r\right)}|a(y)|\left(\int_{B\left(x_{0}, 2 r\right)}\left|\widetilde{F}_{\gamma}(x, y)\right|^{q} d x\right)^{1 / q} d y \\
& \leq C \int_{B\left(x_{0}, r\right)}|a(y)| \rho\left(x_{0}\right)^{-\delta}\left(\int_{B\left(x_{0}, 2 r\right)} \frac{d x}{|x-y|^{(n-\delta) q}}\right)^{1 / q} d y \\
& \leq C \rho\left(x_{0}\right)^{-\delta} r^{n-n / p}\left(\int_{B(0,3 r)} \frac{d x}{|x|^{(n-\delta) q}}\right)^{1 / q} \\
& \leq C \rho\left(x_{0}\right)^{-\delta} r^{n / q-n / p+\delta} \\
& \leq C \rho\left(x_{0}\right)^{n(a-b)} .
\end{aligned}
$$

Here we choose $q$ such that $1<q<n /(n-\delta)$ and $n / q-$ $n / p+\delta>0$ or, equivalently, $1<q<n p /(n-p \delta)$. When $2 r \leq\left|x-x_{0}\right|<2 \rho\left(x_{0}\right)$, using the cancelation condition of $a$ and Lemma 13, we obtain

$$
\begin{aligned}
& \left|\left(L^{i \gamma}-(-\Delta)^{i \gamma}\right) a(x)\right| \\
& \quad=\left|\int_{B\left(x_{0}, r\right)}\left(\widetilde{F}_{\gamma}(x, y)-\widetilde{F}_{\gamma}\left(x, x_{0}\right)\right) a(y) d y\right| \\
& \quad \leq \frac{C \rho\left(x_{0}\right)^{-\delta}}{\left|x-x_{0}\right|^{n}} \int_{B\left(x_{0}, r\right)}|a(y)|\left|y-x_{0}\right|^{\delta} d y \\
& \quad \leq \frac{C \rho\left(x_{0}\right)^{-\delta} r^{n-n / p+\delta}}{\left|x-x_{0}\right|^{n}} .
\end{aligned}
$$


It follows that

$$
\begin{aligned}
J_{2} & \leq C \rho\left(x_{0}\right)^{-\delta} r^{n-n / p+\delta}\left(\int_{2 r \leq\left|x-x_{0}\right|<2 \rho\left(x_{0}\right)} \frac{d x}{\left|x-x_{0}\right|^{n q}}\right)^{1 / q} \\
& \leq C \rho\left(x_{0}\right)^{-\delta} r^{n / q-n / p+\delta} \\
& \leq C \rho\left(x_{0}\right)^{n(a-b)} .
\end{aligned}
$$

When $\left|x-x_{0}\right| \geq 2 \rho\left(x_{0}\right)$, by (29), we have

$$
\begin{aligned}
& \left|\left(L^{i \gamma}-(-\Delta)^{i \gamma}\right) a(x)\right| \\
& \quad=\left|\int_{B\left(x_{0}, r\right)}\left(\widetilde{F}_{\gamma}(x, y)-\widetilde{F}_{\gamma}\left(x, x_{0}\right)\right) a(y) d y\right| \\
& \quad \leq \frac{C}{\left|x-x_{0}\right|^{n+\delta}} \int_{B\left(x_{0}, r\right)}|a(y)|\left|y-x_{0}\right|^{\delta} d y \\
& \quad \leq \frac{C r^{n-n / p+\delta}}{\left|x-x_{0}\right|^{n+\delta}} .
\end{aligned}
$$

Then

$$
\begin{aligned}
J_{3} & \leq C r^{n-n / p+\delta}\left(\int_{\left|x-x_{0}\right| \geq 2 \rho\left(x_{0}\right)} \frac{d x}{\left|x-x_{0}\right|^{(n+\delta) q}}\right)^{1 / q} \\
& \leq C r^{n-n / p+\delta} \rho\left(x_{0}\right)^{-n / q^{\prime}-\delta} \\
& \leq C \rho\left(x_{0}\right)^{n(a-b)} .
\end{aligned}
$$

We have seen that

$$
\left\|\left(L^{i \gamma}-(-\Delta)^{i \gamma}\right) a(x)\right\|_{L^{q}}^{1 /(a-b)} \geq \frac{1}{C} \rho\left(x_{0}\right)^{n} .
$$

As above, there needs no the cancelation condition. To finish the proof, we only need to prove $\mathcal{N}\left(\left(L^{i \gamma}-(-\Delta)^{i \gamma}\right) a\right) \leq C$ or, equivalently,

$$
\left\|\left|x-x_{0}\right|^{n b}\left(L^{i \gamma}-(-\Delta)^{i \gamma}\right) a(x)\right\|_{L^{q}} \leq C \rho\left(x_{0}\right)^{n a} .
$$

Write

$$
\begin{aligned}
& \left(\int_{\mathbb{R}^{n}}\left|x-x_{0}\right|^{n b q}\left|L^{i \gamma}-(-\Delta)^{i \gamma} a(x)\right|^{q} d x\right)^{1 / q} \\
& \leq\left(\int_{B\left(x_{0}, 2 \rho\left(x_{0}\right)\right)}\left|x-x_{0}\right|^{n b q}\left|L^{i \gamma}-(-\Delta)^{i \gamma} a(x)\right|^{q} d x\right)^{1 / q} \\
& \quad+\left(\int_{\left|x-x_{0}\right| \geq 2 \rho\left(x_{0}\right)}\left|x-x_{0}\right|^{n b q}\left|L^{i \gamma}-(-\Delta)^{i \gamma} a(x)\right|^{q} d x\right)^{1 / q} \\
& =H_{1}+H_{2} .
\end{aligned}
$$

It is clear that

$$
H_{1} \leq C \rho\left(x_{0}\right)^{n b}\left\|L^{i \gamma}-(-\Delta)^{i \gamma} a(x)\right\|_{L^{q}} \leq C \rho\left(x_{0}\right)^{n a} .
$$

By (63),

$$
\begin{aligned}
H_{2} & \leq C r^{n-n / p+\delta}\left(\int_{\left|x-x_{0}\right| \geq 2 \rho\left(x_{0}\right)}\left|x-x_{0}\right|^{(n b-n-\delta) q} d x\right)^{1 / q} \\
& \leq C r^{n-n / p+\delta} \rho\left(x_{0}\right)^{n b-\delta-n / q^{\prime}} \\
& \leq C \rho\left(x_{0}\right)^{n a}
\end{aligned}
$$

where we have taken $\epsilon$ such that $1 / p-1<\epsilon<\delta / n$, which implies that $(n b-n-\delta) q+n<0$. The proof is complete.

\section{5. $H_{L}^{1}$-Boundedness}

In this section we prove the boundedness of $\nabla L^{-1 / 2}$ on $H_{L}^{1}$ when $n / 2<q_{0}<n$.

Let $a(x)$ be an $H_{L}^{1, q}$-atom associated with a ball $B\left(x_{0}, r\right)$ for some suitable $q$. As the above section, if $r \geq \rho\left(x_{0}\right)$, we will prove that $\nabla L^{-1 / 2} a(x)$ is an $H_{L}^{1, q, \epsilon}$-molecule up to a constant factor. If $r<\rho\left(x_{0}\right)$, we will prove that $\left(\nabla L^{-1 / 2}-\right.$ $\left.\nabla(-\Delta)^{-1 / 2}\right) a(x)$ is an $H_{L}^{1, q, \epsilon}$-molecule up to a constant factor for some suitable $\epsilon$. In any case we have $\left\|\nabla L^{-1 / 2} a(x)\right\|_{H_{L}^{p}} \leq C$ uniformly.

Suppose $r \geq \rho\left(x_{0}\right)$. It follows from Lemma 11 that

$$
\left\|\nabla L^{-1 / 2} a(x)\right\|_{L^{q}} \leq C\|a(x)\|_{q} \leq C\left|B\left(x_{0}, r\right)\right|^{a-b},
$$

provide $1<q \leq p_{0}$, where $1 / p_{0}=1 / q_{0}-1 / n, a=\epsilon>$ $0, \quad b=1-1 / q+\epsilon$. Thus there needs no the cancelation condition. Write

$$
\begin{aligned}
& \left(\int_{\mathbb{R}^{n}}\left|x-x_{0}\right|^{n b q}\left|\nabla L^{-1 / 2} a(x)\right|^{q} d x\right)^{1 / q} \\
& \leq\left(\int_{B\left(x_{0}, 2 r\right)}\left|x-x_{0}\right|^{n b q}\left|\nabla L^{-1 / 2} a(x)\right|^{q} d x\right)^{1 / q} \\
& \quad+\left(\int_{\left|x-x_{0}\right| \geq 2 r}\left|x-x_{0}\right|^{n b q}\left|\nabla L^{-1 / 2} a(x)\right|^{q} d x\right)^{1 / q} \\
& =I_{1}+I_{2} .
\end{aligned}
$$

It is obvious that

$$
\begin{aligned}
I_{1} & \leq r^{n b}\left\|\nabla L^{-1 / 2} a(x)\right\|_{L^{q}} \\
& \leq C\left|B\left(x_{0}, r\right)\right|^{b}\|a(x)\|_{L^{q}} \leq C\left|B\left(x_{0}, r\right)\right|^{a} .
\end{aligned}
$$


On the other hand, we have

$$
\begin{aligned}
& I_{2} \leq\left(\int _ { | x - x _ { 0 } | \geq 2 r } \left(\int_{B\left(x_{0}, r\right)}\left|x-x_{0}\right|^{n b}\right.\right. \\
& \left.\left.\quad \times\left|R^{L}(x, y)\right||a(y)|\right)^{q} d x\right)^{1 / q} d y \\
& \leq \int_{B\left(x_{0}, r\right)}|a(y)|\left(\int_{\left|x-x_{0}\right| \geq 2 r}\left|x-x_{0}\right|^{n b q}\right. \\
& \left.\times\left|R^{L}(x, y)\right|^{q} d x\right)^{1 / q} d y \\
& =\int_{B\left(x_{0}, r\right)} G(y)|a(y)| d y .
\end{aligned}
$$

Note that $|x-y| \sim\left|x-x_{0}\right|$ when $\left|x-x_{0}\right| \geq 2 r$ and $y \in B\left(x_{0}, r\right)$ and by Lemma 11,

$$
\begin{aligned}
& G(y) \\
& \leq C \rho(y)^{N} \\
& \quad \times\left\{\left(\int_{\left|x-x_{0}\right| \geq 2 r}\left(\int_{B(x,|x-y| / 4)} \frac{V(z) d z}{|z-x|^{n-1}}\right)^{q}\right.\right. \\
& \left.\quad \times \frac{d x}{\left|x-x_{0}\right|^{(N-n b+n-1) q}}\right)^{1 / q} \\
& \left.+\left(\int_{\left|x-x_{0}\right| \geq 2 r} \frac{d x}{\left|x-x_{0}\right|^{(N-n b+n) q}}\right)^{1 / q}\right\} .
\end{aligned}
$$

Since $\rho(y) \leq C r$ for $y \in B\left(x_{0}, r\right)$, it is clear that

$$
\begin{aligned}
& \rho(y)^{N}\left(\int_{\left|x-x_{0}\right| \geq 2 r} \frac{d x}{\left|x-x_{0}\right|^{(N-n b+n) q}}\right)^{1 / q} \\
& \leq C r^{n b-n / q^{\prime}} \leq C\left|B\left(x_{0}, r\right)\right|^{a}
\end{aligned}
$$

provide $N>$ na. We have taken $q$ such that $1<q \leq p_{0}$, where $1 / p_{0}=1 / q_{0}-1 / n$. Let $1 / q=1 / s-1 / n$. Then $s \leq q_{0}$. Using the theorem on fractional integrals, $B_{s}$ condition, and Lemma 8, we obtain

$$
\begin{aligned}
& \rho(y)^{N}\left(\int_{\left|x-x_{0}\right| \geq 2 r}\left(\int_{B(x,|x-y| / 4)} \frac{V(z) d z}{|z-x|^{n-1}}\right)^{q}\right. \\
&\left.\times \frac{d x}{\left|x-x_{0}\right|^{(N-n b+n-1) q}}\right)^{1 / q} \\
& \leq C^{N} \sum_{j=1}^{\infty}\left(\int_{2^{j} r \leq\left|x-x_{0}\right|<2^{j+1} r} \frac{1}{\left|x-x_{0}\right|^{(n+N-n b-1) q}}\right.
\end{aligned}
$$

$$
\begin{aligned}
& \left.\cdot\left(\int_{B(x,|x-y| / 4)} \frac{V(z) d z}{|z-x|^{n-1}}\right)^{q} d x\right)^{1 / q} \\
\leq & C \sum_{j=1}^{\infty} 2^{-j N}\left(2^{j} r\right)^{-n+n b+1} \\
& \times\left(\int_{B\left(x_{0}, 2^{j+1} r\right)}\left(\int_{B(x,|x-y| / 4)} \frac{V(z) d z}{|z-x|^{n-1}}\right)^{q} d x\right)^{1 / q} \\
\leq & C \sum_{j=1}^{\infty} 2^{-j N}\left(2^{j} r\right)^{-n+n b+1}\left(\int_{B\left(x_{0}, 2^{j+1} r\right)} V(x)^{s} d x\right)^{1 / s} \\
\leq & C \sum_{j=1}^{\infty} 2^{-j N}\left(2^{j} r\right)^{-n+n b+1}\left|B\left(x_{0}, 2^{j+1} r\right)\right|^{1 / s-1} \\
& \times \int_{B\left(x_{0}, 2^{j+1} r\right)} V(x) d x \\
\leq & C \sum_{j=1}^{\infty} 2^{-j\left(N-m_{0}\right)}\left(2^{j} r\right)^{-n+n b+n / s-1} \\
\leq & \left.C \sum_{j=1}^{\infty} 2^{-j\left(N-m_{0}-n a\right)} r^{n a}\left(x_{0}, r\right)\right|^{a}
\end{aligned}
$$

provide $N$ sufficiently large. Thus $G(y) \leq C\left|B\left(x_{0}, r\right)\right|^{a}$. It follows that

$$
\begin{aligned}
I_{2} & \leq \int_{B\left(x_{0}, r\right)} G(y)|a(y)| d y \\
& \leq C\left|B\left(x_{0}, r\right)\right|^{a}\|a\|_{L^{1}} \leq C\left|B\left(x_{0}, r\right)\right|^{a} .
\end{aligned}
$$

Therefore, $\left\|\left|\cdot-x_{0}\right|^{n b} \nabla L^{-1 / 2} a\right\|_{L^{q}} \leq C\left|B\left(x_{0}, r\right)\right|^{a}$ and $\mathcal{N}\left(\nabla L^{-1 / 2} a\right) \leq C$.

In case $r<\rho\left(x_{0}\right)$, we need to prove that $\left(\nabla L^{-1 / 2}-\right.$ $\left.\nabla(-\Delta)^{-1 / 2}\right) a(x)$ is an $H_{L}^{1, q, \epsilon}$-molecule up to a constant factor for some suitable $\epsilon$. First we give the estimate of $\left\|\left(\nabla L^{-1 / 2}-\nabla(-\Delta)^{-1 / 2}\right) a(x)\right\|_{L^{q}}$. Write

$$
\begin{aligned}
& \left(\int_{\mathbb{R}^{n}}\left|\left(\nabla L^{-1 / 2}-\nabla(-\Delta)^{-1 / 2}\right) a(x)\right|^{q} d x\right)^{1 / q} \\
& \leq\left(\int_{B\left(x_{0}, 2 \rho\left(x_{0}\right)\right)}\left|\left(\nabla L^{-1 / 2}-\nabla(-\Delta)^{-1 / 2}\right) a(x)\right|^{q} d x\right)^{1 / q} \\
& \quad+\left(\int_{\left|x-x_{0}\right| \geq 2 \rho\left(x_{0}\right)}\left|\left(\nabla L^{-1 / 2}-\nabla(-\Delta)^{-1 / 2}\right) a(x)\right|^{q} d x\right)^{1 / q} \\
& =J_{1}+J_{2} .
\end{aligned}
$$


We have

$$
\begin{aligned}
J_{1} & \leq \int_{B\left(x_{0}, r\right)}|a(y)|\left(\int_{B\left(x_{0}, 2 \rho\left(x_{0}\right)\right)}|\widetilde{R}(x, y)|^{q} d x\right)^{1 / q} d y \\
& =\int_{B\left(x_{0}, r\right)} \widetilde{G}(y)|a(y)| d y .
\end{aligned}
$$

By Lemma 11,

$$
\begin{aligned}
& \widetilde{G}(y) \leq\left(\int_{B\left(x_{0}, 2 \rho\left(x_{0}\right)\right)} \frac{C}{|x-y|^{(n-1) q}}\right. \\
&\left.\times\left(\int_{B(x,|x-y| / 4)} \frac{V(z) d z}{|z-x|^{n-1}}\right)^{q} d x\right)^{1 / q} \\
&+C \rho(y)^{-\delta}\left(\int_{B\left(x_{0}, 2 \rho\left(x_{0}\right)\right)} \frac{d x}{|x-y|^{(n-\delta) q}}\right)^{1 / q} \\
&=\widetilde{G}_{1}(y)+\widetilde{G}_{2}(y) .
\end{aligned}
$$

Note that $\rho(y) \sim \rho\left(x_{0}\right)$ when $y \in B\left(x_{0}, r\right)$. It is obvious that

$$
\widetilde{G}_{2}(y) \leq C \rho\left(x_{0}\right)^{-n / q^{\prime}}=C \rho\left(x_{0}\right)^{n(a-b)},
$$

provide $1<q<n /(n-\delta)$. On the other hand, using the theorem on fractional integrals and $B_{s}$ condition with $s \leq$ $q_{0}, 1 / q=1 / s-1 / n$, and $1<q<n /(n-\delta)$, we get

$$
\begin{aligned}
& \widetilde{G}_{1}(y) \leq \sum_{j=0}^{\infty}\left(\int_{2^{-j+1} \rho\left(x_{0}\right) \leq|x-y|<2^{-j+2} \rho\left(x_{0}\right)} \frac{C}{|x-y|^{(n-1) q}}\right. \\
& \left.\left(\int_{B(x,|x-y| / 4)} \frac{V(z) d z}{|z-x|^{n-1}}\right)^{q} d x\right)^{1 / q} \\
& \leq C \sum_{j=0}^{\infty}\left(2^{-j} \rho\left(x_{0}\right)\right)^{-n+1} \\
& \times\left(\int_{|x-y|<2^{-j+2} \rho\left(x_{0}\right)}\right. \\
& \left.\times\left(\int_{B(x,|x-y| / 4)} \frac{V(z) d z}{|z-x|^{n-1}}\right)^{q} d x\right)^{1 / q} \\
& \leq C \sum_{j=0}^{\infty}\left(2^{-j} \rho\left(x_{0}\right)\right)^{-n+1}\left(\int_{|x-y|<2^{-j+3} \rho\left(x_{0}\right)} V(x)^{s} d x\right)^{1 / s} \\
& \leq C \sum_{j=0}^{\infty}\left(2^{-j} \rho\left(x_{0}\right)\right)^{-2 n+n / s+1} \int_{|x-y|<2^{-j+3} \rho\left(x_{0}\right)} V(x) d x \\
& \leq C \sum_{j=0}^{\infty}\left(2^{-j} \rho\left(x_{0}\right)\right)^{-n+n / s-1} 2^{-j \delta} \\
& \leq C \rho\left(x_{0}\right)^{n(a-b)},
\end{aligned}
$$

where we have used Lemma 7 in the last second inequality. Thus,

$$
J_{1} \leq \int_{B\left(x_{0}, r\right)} \widetilde{G}(y)|a(y)| d y \leq C \rho\left(x_{0}\right)^{n(a-b)} .
$$

Since $|x-y| \sim\left|x-x_{0}\right|$ when $\left|x-x_{0}\right| \geq 2 \rho\left(x_{0}\right)$ and $y \in B\left(x_{0}, r\right)$, $|\widetilde{R}(x, y)| \leq C /|x-y|^{n}$, it is easy to see that

$$
\begin{aligned}
J_{2} & \leq \int_{B\left(x_{0}, r\right)}|a(y)|\left(\int_{\left|x-x_{0}\right| \geq 2 \rho\left(x_{0}\right)}|\widetilde{R}(x, y)|^{q} d x\right)^{1 / q} d y \\
& \leq C \int_{B\left(x_{0}, r\right)}|a(y)|\left(\int_{\left|x-x_{0}\right| \geq 2 \rho\left(x_{0}\right)} \frac{d x}{|x-y|^{n q}}\right)^{1 / q} d y \\
& \leq C \int_{B\left(x_{0}, r\right)}|a(y)| \rho\left(x_{0}\right)^{n(a-b)} d y \\
& \leq C \rho\left(x_{0}\right)^{n(a-b)} .
\end{aligned}
$$

Therefore we have

$$
\left\|\left(\nabla L^{-1 / 2}-\nabla(-\Delta)^{-1 / 2}\right) a(x)\right\|_{L^{q}} \leq C \rho\left(x_{0}\right)^{n(a-b)} .
$$

As above, there needs no the cancelation condition. Write

$$
\begin{aligned}
& \left(\int_{\mathbb{R}^{n}}\left|x-x_{0}\right|^{n b q}\left|\left(\nabla L^{-1 / 2}-\nabla(-\Delta)^{-1 / 2}\right) a(x)\right|^{q} d x\right)^{1 / q} \\
& \leq\left(\int_{B\left(x_{0}, 2 \rho\left(x_{0}\right)\right)}\left|x-x_{0}\right|^{n b q}\right. \\
& \left.\quad \times\left|\left(\nabla L^{-1 / 2}-\nabla(-\Delta)^{-1 / 2}\right) a(x)\right|^{q} d x\right)^{1 / q} \\
& \quad+\left(\int_{\left|x-x_{0}\right| \geq 2 \rho\left(x_{0}\right)}\left|x-x_{0}\right|^{n b q}\left|\nabla L^{-1 / 2} a(x)\right|^{q} d x\right)^{1 / q} \\
& \quad+\left(\int_{\left|x-x_{0}\right| \geq 2 \rho\left(x_{0}\right)}\left|x-x_{0}\right|^{n b q}\left|\nabla(-\Delta)^{-1 / 2} a(x)\right|^{q} d x\right)^{1 / q} \\
& =H_{1}+H_{2}+H_{3} .
\end{aligned}
$$

It is obvious that

$$
H_{1} \leq C \rho\left(x_{0}\right)^{n b}\left\|\left(\nabla L^{-1 / 2}-\nabla(-\Delta)^{-1 / 2}\right) a(x)\right\|_{L^{q}} \leq C \rho\left(x_{0}\right)^{n a} .
$$

We have

$$
\begin{aligned}
& H_{2} \leq \int_{B\left(x_{0}, r\right)}|a(y)| \\
& \times\left(\int_{\left|x-x_{0}\right| \geq 2 \rho\left(x_{0}\right)}\left|x-x_{0}\right|^{n b q}\right. \\
& \left.\times\left|R^{L}(x, y)\right|^{q} d x\right)^{1 / q} d y \\
& =\int_{B\left(x_{0}, r\right)} G_{0}(y)|a(y)| d y .
\end{aligned}
$$


Since $|x-y| \sim\left|x-x_{0}\right|$ and $\rho(y) \sim \rho\left(x_{0}\right)$ when $\left|x-x_{0}\right| \geq$ $2 \rho\left(x_{0}\right)$ and $y \in B\left(x_{0}, r\right)$, by Lemma 11 ,

$$
\begin{aligned}
& G_{0}(y) \\
& \leq C \rho\left(x_{0}\right)^{N} \\
& \quad \times\left\{\left(\int_{\left|x-x_{0}\right| \geq 2 \rho\left(x_{0}\right)}\left(\int_{B(x,|x-y| / 4)} \frac{V(z) d z}{|z-x|^{n-1}}\right)^{q}\right.\right. \\
& \left.\left.\left.\quad \times \frac{d x}{\left|x-x_{0}\right|^{(N-n b+n-1) q}}\right)^{1 / q}\right)^{1 / q}\right\} . \\
& +\left(\int_{\left|x-x_{0}\right| \geq 2 \rho\left(x_{0}\right)} \frac{d x}{\left|x-x_{0}\right|^{(N-n b+n) q}}\right)^{1 / q}
\end{aligned}
$$

Similar to $G(y)$ in the proof of (77), we obtain $G_{0}(y) \leq$ $C \rho\left(x_{0}\right)^{n a}$ by the same argument. It follows that

$$
H_{2} \leq \int_{B\left(x_{0}, r\right)} G_{0}(y)|a(y)| d y \leq C \rho\left(x_{0}\right)^{n a} .
$$

Using the cancelation condition of $a$,

$$
\begin{aligned}
H_{3} \leq \int_{B\left(x_{0}, r\right)}|a(y)|\left(\int_{\left|x-x_{0}\right| \geq 2 \rho\left(x_{0}\right)}\left|x-x_{0}\right|^{n b q}\right. \\
\left.\quad \times\left|R(x, y)-R\left(x, x_{0}\right)\right|^{q} d x\right)^{1 / q} d y \\
\leq C \int_{B\left(x_{0}, r\right)}|a(y)| \\
\quad \times\left(\int_{\left|x-x_{0}\right| \geq 2 \rho\left(x_{0}\right)} \frac{\left|y-x_{0}\right|^{q} d x}{\left|x-x_{0}\right|^{(n+1-n b) q}}\right)^{1 / q} d y \\
\leq C \int_{B\left(x_{0}, r\right)}|a(y)| \rho\left(x_{0}\right)^{n a} d y \\
\leq C \rho\left(x_{0}\right)^{n a},
\end{aligned}
$$

where we have taken $\epsilon$ such that $0<\epsilon<1 / n$. This proves that

$$
\left\|\left|x-x_{0}\right|^{n b}\left(\nabla L^{-1 / 2}-\nabla(-\Delta)^{-1 / 2}\right) a(x)\right\|_{L^{q}} \leq C \rho\left(x_{0}\right)^{n a} .
$$

It follows that $\mathscr{N}\left(\left(\nabla L^{-1 / 2}-\nabla(-\Delta)^{-1 / 2}\right) a\right) \leq C$. The proof is completed.

\section{Conflict of Interests}

The authors declare that there is no conflict of interests regarding the publication of this paper.

\section{Acknowledgments}

This work was supported by Research Fund for the Doctoral Program of Higher Education of China (20113108120001), the
National Natural Science Foundation of China under Grants nos. 11471018 and 11371036, and the Beijing Natural Science Foundation under Grant no. 1142005.

\section{References}

[1] Z. W. Shen, " $L^{p}$ estimates for $L^{p}$ operators with certain potentials," Annales de l'Institut Fourier, vol. 45, no. 2, pp. 513-546, 1995.

[2] C. L. Fefferman, "The uncertainty principle," American Mathematical Society, vol. 9, no. 2, pp. 129-206, 1983.

[3] J. Zhong, Harmornic analysis for some Schrödinger type operators [Ph.D. thesis], Princeton University, 1993.

[4] J. Dziubański and J. Zienkiewicz, "Hardy space $H^{1}$ associated to Schrödinger operator with potential satisfying reverse Hölder inequality," Revista Matemática Iberoamericana, vol. 15, pp. 279-296, 1999.

[5] J. Dziubański and J. Zienkiewicz, " $H^{p}$ spaces for Schrödinger operators, Fourier Analysis and Ralated Topics," Banach Center Publications, vol. 56, pp. 45-53, 2002.

[6] J. Dziubański and J. Zienkiewicz, " $H^{P}$ spaces associated with Schrödinger operators with potentials from reverse Hölder classes," Colloquium Mathematicum, vol. 98, no. 1, pp. 5-38, 2003.

[7] J. Z. Huang and H. P. Liu, "Littlewood-Paley theory and Hardy spaces related to Schrödinger operators," Submitted.

[8] M. Taibleson and G. Weiss, "The molecular characterization of certain Hardy spces," Asterisque, vol. 77, pp. 67-151, 1980.

[9] J. Alvarez and M. Milman, " $H^{p}$ continuty properties of Calderon-Zygmund operators," Journal of Mathematical Anal$y$ sis and Applications, vol. 118, pp. 65-79, 1986. 


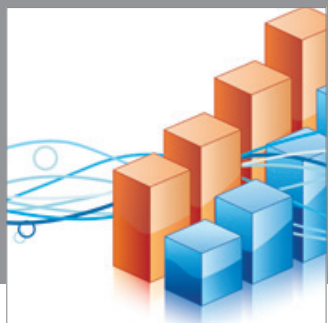

Advances in

Operations Research

mansans

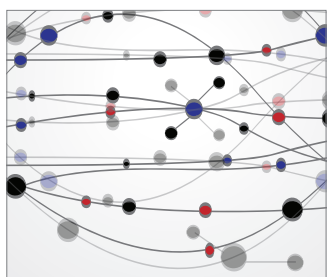

The Scientific World Journal
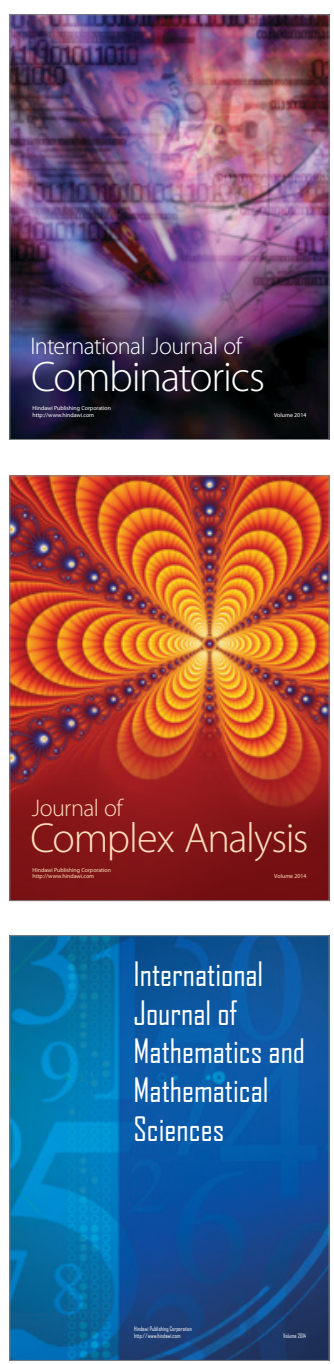
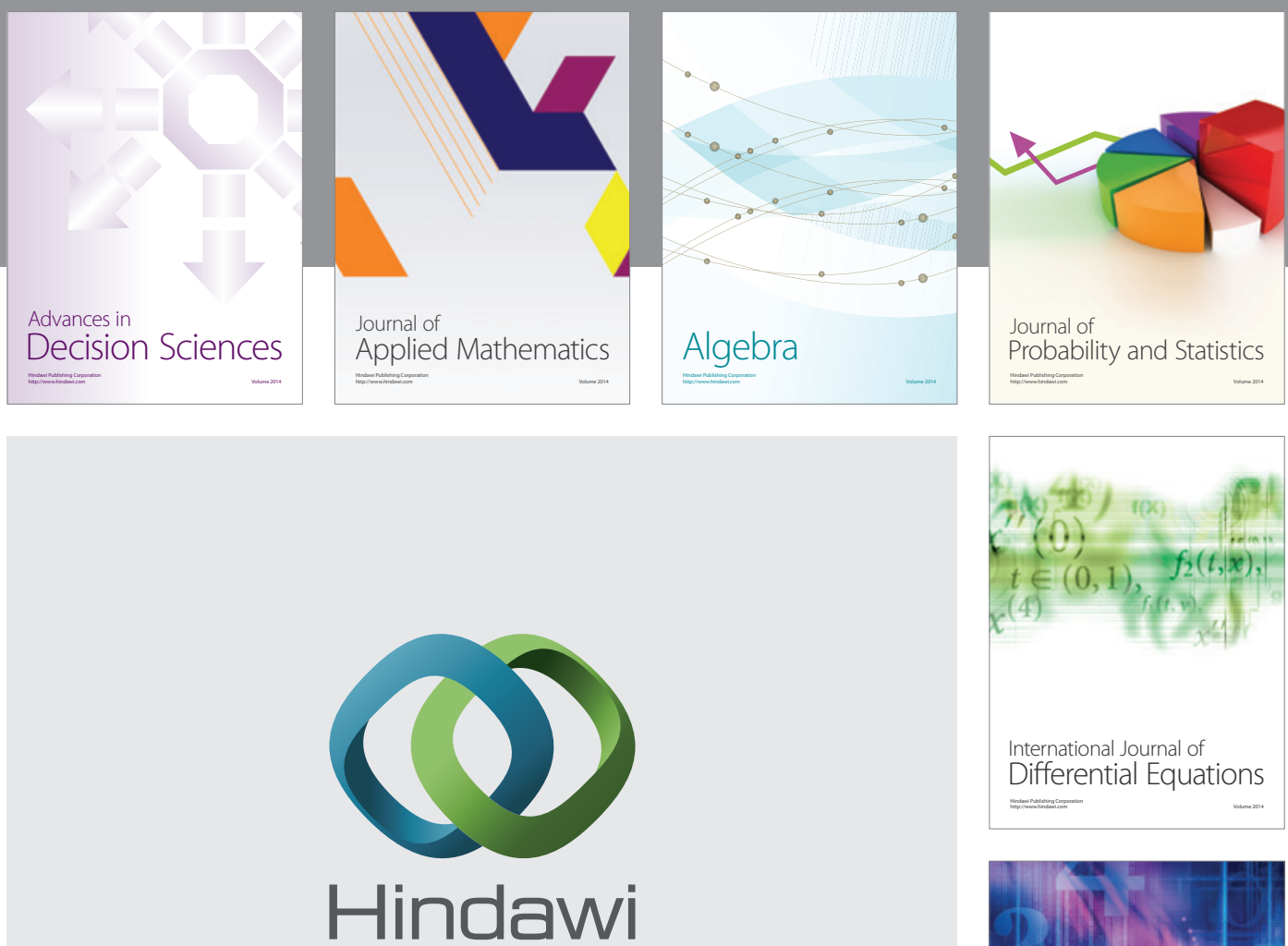

Submit your manuscripts at http://www.hindawi.com
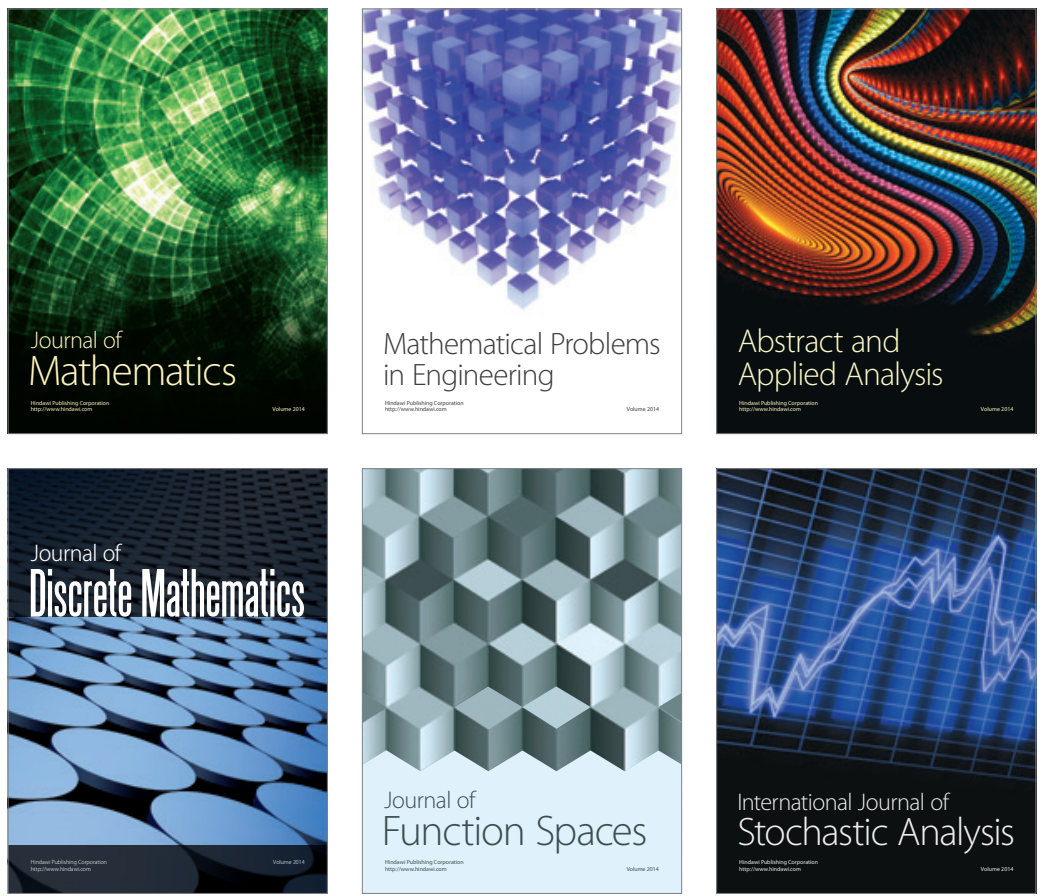

Journal of

Function Spaces

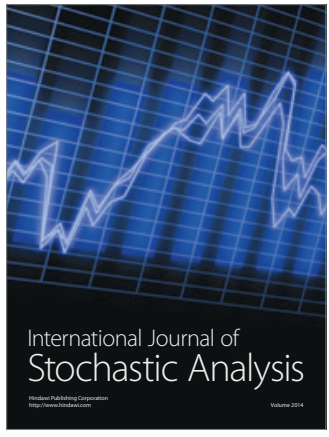

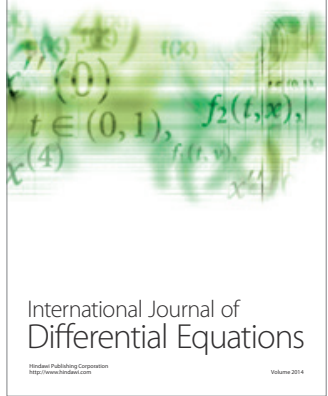
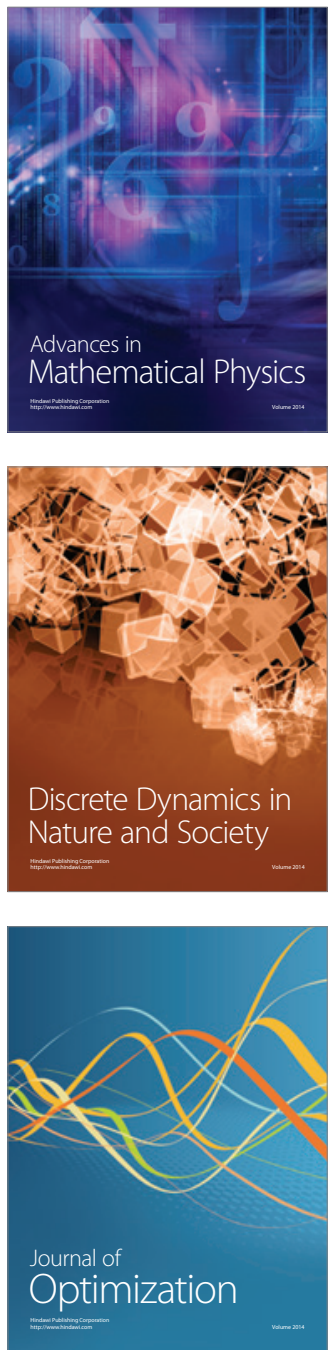